\title{
An Initial Investigation of Disrupted Intracortical Myelin as a Novel Brain Marker of Alcohol Use Disorder
}

Vanessa Morris, BA ${ }^{1}$, Nicholas Bock, $\mathrm{PhD}^{2}$, Luciano Minuzzi, $\mathrm{PhD}^{3}$, James MacKillop, $\mathrm{PhD}^{4}$, Michael Amlung, $\mathrm{PhD}^{5}$

\section{Affiliations:}

1. Peter Boris Center for Addictions Research; McMaster University

2. Department of Psychology, Neuroscience, and Behaviour; McMaster University

3. Department of Psychiatry and Behavioural Neurosciences, McMaster University

4. Peter Boris Center for Addictions Research; McMaster University

5. Cofrin Logan Center for Addiction Research and Treatment, University of Kansas; Peter Boris Center for Addictions Research, McMaster University

Location the Work Was Carried Out: 100 West $5^{\text {th }}$ Street, Hamilton Ontario, L8P 3R2

Correspondence: Michael Amlung, PhD. Cofrin-Logan Center for Addiction Research and Treatment, University of Kansas, 1000 Sunnyside Ave, Lawrence, KS 66045; mamlung@ku.edu 


\begin{abstract}
Although disruption of cortical gray matter and white matter tracts are well-established markers of alcohol use disorder (AUD), this is the first study to examine the specific role of intracortical myelin (ICM; i.e., highly myelinated gray matter in deeper cortical layers) in AUD. The current study used a 3T MRI sequence optimized for high intracortical contrast to examine patterns of ICM-related MRI signal in 30 individuals with AUD and 33 healthy social drinkers. Secondary aims included exploring continuous associations with alcohol problem severity and examining sex differences. Surface-based analytic techniques were used to quantify ICMrelated MRI signal for a priori region of interest analyses (20 bilateral regions) and exploratory vertex-wise analyses (using Cohen's d). Although the distribution of ICM-related signal was generally comparable between groups, the AUD group exhibited significantly $(p<.05)$ greater ICM-related MRI signal in precuneus, ventromedial prefrontal cortex, posterior cingulate, middle anterior cingulate, middle/posterior insula, dorsolateral prefrontal cortex, and posterior cingulate, among other regions (Cohen's $d=.50-.75$, indicating medium magnitude effects). Significant positive correlations between ICM signal and AUD severity were found in several frontal, parietal, cingulate, and temporal regions (rs .25-.34). No sex differences in ICM were observed. These findings provide initial proof-of-concept for examining ICM in relation to AUD. Understanding the pathophysiological mechanisms of these associations (e.g., neuroinflammation) and the clinical relevance of ICM is warranted.
\end{abstract}




\section{Introduction}

Structural and functional disruptions of the cerebral cortex are well-established indicators of alcohol use disorder (AUD) [1,2]. People who meet criteria for AUD have been found to exhibit reduced cortical thickness [3,4], reduced white and grey matter volume [5], as well as

reduced volume in the frontal cortex and other cortical and sub-cortical regions $[3,6,7]$ relative to people without an AUD. Cortical thickness deficits are related to a number of clinical indicators in AUD, including associations with degree of alcohol use and misuse [4,8], poor AUD treatment outcomes [3], and impaired inhibitory control in people with AUD [9]. Although it has been theorized that the brain reaches peak development at roughly twenty-five years of age [10], some literature suggests that white matter may have a more prolonged trajectory $[11,12]$. Various studies have found that while grey matter may peak in one's mid-twenties, heavilymyelinated white matter on the other hand, may not fully develop until the third, fourth, or fifth decade of one's life $[11,13]$. As a result of this deferred development, white matter remains inherently sensitive and susceptible to damage from the use of addictive substances, environmental effects, and physical insults [14]. Reasons for the extended vulnerability in white matter include its low level of blood supply $[11,12,14]$ and the extensive energy requirements of oligodendrocyte function $[11,12,14]$. Increased focus on studying associations between brain myelination and substance use disorders is an important and clinically-significant priority.

Although cortical deficits in AUD are generally well-established, the cerebral cortex is highly heterogenous with respect to types of neuronal tissue [15]. In particular, there is growing interest in characterizing the role of myelin in the cortex. Myelin serves many functions, including increasing action potential transmission speed, improving neuronal synchrony, increasing brain connectivity, decreasing refractory time, and supporting cognitive functions [11]. Although primarily concentrated in neuronal tracts in the white matter, myelinated axons are also found in the cortex, with deeper layers (IV-VI) being more heavily myelinated than the superficial layers (I-III) [16]. These deeper, myelinated layers of the cortex represent what has 
been termed intracortical myelin (ICM) [16]. Similar to myelin in white matter, ICM reaches peak development between the third to fifth decade of one's life $[11,13]$ and thus remains vulnerable to toxins and damage $[11,17]$. Intracortical myelin is thought to play a central role in synchronizing and speeding of neuronal signals through the cortex in support of cognitive processing. For example, research has shown that decreased ICM in posterior cingulate cortex in healthy individuals is correlated with decreased error processing and cognitive control [18] and reduced ICM in insula and superior temporal gyrus is associated with increased performance variability [19].

Since the magnetic resonance imaging (MRI) signal is very sensitive to the presence of myelin, several MRI protocols have been developed to image ICM content in vivo [20,21]. In the protocol developed by Bock et al. [21], the T1-weighted MRI contrast was optimized for differentiating cortical regions with low and high myelin content. This T1 image is commonly divided by a proton-density-weighted image to generate a ratio image that is strongly $\mathrm{T} 1$ weighted with high intracortical contrast. Image processing tools are then applied to generate a whole-brain map of the ICM ratio signal that is projected on the middle-depth cortical surface. Various studies have confirmed these in vivo MRI measurements of ICM by comparing the MRI maps to histological samples of nonhuman primate brain tissue [22], as well as samples of postmortem human brain tissue [23]. The results of these histological studies confirm that MRIgenerated ICM maps provide an accurate representation of underlying myeloarchitecture across the cortex.

The MRI protocol developed by Bock et al. has been used in multiple studies of ICM in healthy $[16,24]$ and clinical populations $[16,24,25]$. For example, it has been suggested that disruptions in ICM may partially underlie susceptibility to the development of mental health disorders such as bipolar disorder and schizophrenia [11]. Rowley et al., (2015) used MRI with high intracortical contrast in a sample of healthy adult females and female participants with bipolar disorder. They examined total cortical thickness, as well as myelinated (ICM) and 
unmyelinated cortical thicknesses. The healthy participants displayed the greatest degree of myelination in the precentral gyrus, postcentral gyrus, Heschel's gyrus, posterior cingulate gyrus, as well as visual cortex, which is consistent with other research demonstrating that the human motor cortex (located on the precentral gyrus) displays the highest degree of myelinated fiber density [26]. The comparison of healthy participants to those with bipolar disorder revealed that total cortical thickness was significantly reduced in the bipolar disorder group, and that there was a trend towards less cortical myelination in the bipolar disorder group [16]. Additional research examining white matter in bipolar disorder has found that patients have fewer oligodendrocytes, reduced myelin-related genes, and significant age-related deficits in ICM development [27-30]. Moreover, impairments in neurocognitive performance (i.e., verbal memory) have been associated with ICM in patients with bipolar disorder [25]. In the context of schizophrenia, ICM has been shown dysregulated in the frontal lobes [31]. More recently, Tishler et al. [32] found that patients with schizophrenia displayed a significantly different agerelated ICM trajectory compared to healthy controls.

The studies reviewed above suggest that ICM may be an important neural marker of psychiatric disorders; however there has yet to be a study examining ICM in addictive disorders even though there is evidence to suggest that myelin and ICM are inherently sensitive and vulnerable to neurotoxins, such as alcohol [14]. One study found that mice subjected to maternal binge-drinking in utero, exhibited reductions in multiple myelin-related proteins that were associated with various neurocognitive impairments (motor coordination, spatial awareness, etc.) [33]. As mentioned previously, there is a variety of evidence demonstrating cortical thickness and brain volume deficits in AUD, but these studies have largely examined indices of total cortical thickness or gray matter volume which do not take into account the rich neurobiology of the cortex. Fortunately, the ability to image ICM-related signal in vivo with the optimized MRI pulse sequences described above affords a unique opportunity to explore 
differential effects in cortical sub-units. This research may elucidate whether the overall cortical reductions and disruptions are attributable its myelin content.

The current study examined ICM in adults with AUD compared to social drinkers without AUD using the Bock et al. [21] optimized MRI protocol for imaging ICM in vivo and a postprocessing protocol for investigating changes over the cortical surface [34]. Being the first study to examine ICM in relation to AUD, we refrained from composing a directional hypothesis and instead assumed that there would be some degree of change in ICM signal between the two groups. We also examined continuous associations between ICM and alcohol problem severity as assessed by the Alcohol Use Disorders Identification Test (AUDIT) questionnaire [35]. We selected nine bilateral a priori regions of interest (ROIs) based on theoretical models implicating frontal lobe circuitry [36-38], cortical thickness findings from previous AUD studies [3,4,8,3941], and studies of neurocognitive correlates of ICM $[18,19]$. The ROls examined included: middle frontal gyrus (MFG), superior frontal gyrus (SFG), inferior frontal gyrus (IFG), anterior cingulate (ACC), posterior cingulate (PCC), anterior insula, superior temporal gyrus (STG), middle temporal gyrus (MTG), and precuneus, orbital frontal gyrus (OFG), and dorsolateral prefrontal cortex (DLPFC). A whole-brain vertex-wise analysis examined effect size differences outside of the a priori ROIs. An exploratory aim of the current study was to examine ICM differences between male and female participants. A number of studies have identified differences between males and females with addictive disorders in relation to epidemiology, treatment outcomes, and effects on brain structure and function (for review see McHugh, Votaw, Sugarman, \& Greenfield, 2018). Significant sex disparity still exists in structural brain imaging studies, with many studies not routinely reporting sex differences.

\section{Materials and Methods}

\section{Participants}

Participants were recruited from the Hamilton, Ontario, Canada community including four addiction treatment centers in the region. All participants had to be between the ages of 25-55, 
have no contraindications against MRI, no history of severe head or brain trauma with loss of consciousness, neurological disorder, or severe psychiatric disorder (schizophrenia, bipolar disorder, post-traumatic stress disorder), and meet criteria for current AUD or non-hazardous social drinking. Specifically, participants in the AUD group $(N=30)$ met DSM-5 criteria for AUD and did not meet DSM-5 for any substance use disorder other than nicotine dependence. Participants in the social drinker (control) group $(N=33)$ reported drinking on at least a weekly basis, but did not exceed NIAAA weekly drinking limits of $\geq 14 / 7$ drinks per week for males/females and did not meet DSM-5 criteria for AUD or SUD, except for nicotine dependence. For full demographics, please see Table 1.

\section{Procedures}

The Hamilton Integrated Research Ethics Board approved the study (Project \#1747) and participants provided informed consent. Individuals who were interested in participating were first screened to determine their eligibility. Individuals who met all eligibility criteria were then scheduled for the first of two in-person sessions. The first session took place at our laboratory or at the participant's respective treatment centers. During the first session, participants completed a battery of self-report questionnaires, a clinical interview, and neurocognitive measures. Upon completion of the first session, participants received a $\$ 25$ gift card to local stores. Those who were eligible and interested in continuing were then scheduled for an MRI at the Imaging Research Centre at St. Joseph's Healthcare Hamilton. All participants completed MRI safety screening with the MRI technologist. The MRI scan lasted roughly 40 minutes, after which participants were debriefed, received a $\$ 25$ gift card, and the session concluded.

\section{Measures}

Participants completed a variety of clinical and individual differences measures during the first session. These measures were administered either on the computer, one-on-one with a researcher, or individually using paper and pencil forms. 
Structured Clinical Interview DSM-5 (SCID-5)

Diagnoses of current (i.e., in last 12 months) alcohol use disorder and substance use disorder were obtained via the Structured Clinical Interview for DSM-5 (SCID-5; First, Williams, Karg, \& Spitzer, 2015). All participants completed the alcohol use disorder module and participants who reported using drugs also completed the substance use disorder module for their most often used substance.

\section{Alcohol-related Problems}

The AUDIT [35] was used as a self-report measure of drinking behavior and alcoholrelated problems. The AUDIT is a 10-item self-report scale with total scores ranging from 0-40. Scores of 8 or greater are typically considered to be indicative of hazardous alcohol drinking. Alcohol and Substance Use Frequency

The timeline follow-back interview was used to assess participants' daily alcohol consumption on for the thirty days prior to the assessment [44]. The primary dependent measure was the number of drinks consumed per week, however, given that 27 of 30 participants in the AUD group were recruited from treatment centers, there was a high percentage of participants who reported no drinking in the last 30 days. Thus, the drinks per week variable was for descriptive purposes only and not used in the primary analyses. The modified version of the National Institute on Drug Abuse Alcohol, Smoking and Substance Involvement Screening Test (NIDA Modified ASSIST, https://www.drugabuse.gov/sites/default/files/pdf/nmassist.pdf) assessed frequency of use of ten substances (cannabis, cocaine, prescription stimulants, methamphetamine, inhalants, sedatives, hallucinogens, street opioids, and prescription opioids). Participants rated their use in the past three months, with responses ranging from Never to Multiple Times Daily.

\section{Nicotine Dependence}


Participants who reported current smoking completed the Fagerström Test for Nicotine Dependence (FTND; [45], a validated self-report assessment of cigarette consumption and nicotine dependence severity.

\section{Demographics}

A demographic questionnaire asked participants to provide information on their age, sex assigned at birth, ethnicity, years of education, smoking status, income, and handedness.

\section{Imaging Methods}

Image Acquisition

MRI images were acquired on a 3T General Electric Discovery scanner using a 32channel receive-only radio frequency coil for the head and a transmit radio frequency body coil. All images were acquired with $1 \mathrm{~mm}$ isotropic resolution using a scan protocol developed by Bock and colleagues [21] and used in several prior studies of ICM [16,24,25]. Full details of the MRI scanning protocol are provided in Supplementary Materials. The total scanning protocol was roughly 40 minutes.

Image Preprocessing and Data Analysis

Image processing was performed predominately in FSL (v6.0.0), Freesurfer (v5.3.0) and Connectome Workbench (Human Connectome Project, v1.3.2). Preprocessing and ICM quantification closely followed published procedures [16,24,34]. The complete preprocessing pipeline is described in Supplementary Materials. Briefly, preprocessing included motion correction, registration to $\mathrm{MNI}$ space, spatial smoothing $(5 \times 5 \times 5 \mathrm{~mm} 3 \mathrm{D}$ median filter). A strongly $T_{1}$-weighted ratio image was created by dividing the $T_{1}$-weighted high intracortical contrast image by the filtered proton-density-weighted image. This removed radiofrequency receive field $\left(B_{1}-\right)$ inhomogeneities, some radiofrequency transmit field $\left(B_{1+}\right)$ inhomogeneities, and $T_{2}{ }^{*}$ weighting arising from the gradient echo readout in the inversion-recovery image. Freesurfer segmentation was performed using recon-all. The remaining processing steps closely followed the Human Connectome Project's minimal processing pipeline using custom scripts for myelin 
mapping adapted from the HCP scripts [34]. Each participant's ratio image signal is projected onto the $1 / 2$ cortical depth surface (HCP midthickness surface) using Matlab's SurfStat toolbox.

Group comparisons were performed using an a priori anatomical ROI approach and a whole-brain vertex-wise analysis. ROls were generated using the multi-modal parcellation atlas (MMP) [46]. To reduce inflation of type-1 error due to many small regions, we consolidated neighboring ROls into composite regions corresponding to our a priori ROls. The $40 \mathrm{ROIs}$ examined (20 symmetrical regions per hemisphere) are provided in Table 2 and anatomical locations are visualized in Figure 1 (see Supplementary Table 1 for MPP region numbers).

The mean ICM signal within each ROI was extracted and exported to SPSS Version 26 for group analyses using analysis of covariance models. Covariates were selected based on a two-step process. Demographic and substance use variables that differed significantly between groups were subsequently examined in bivariate correlations with overall ICM-related ratio signal (sum of 20 ROls per hemisphere). Only covariates that were significantly correlated at $p<.05$ were included. An exploratory vertex-wise analysis was conducted by calculating effect size (Cohen's d) for differences between groups at each vertex. Finally, sex differences were explored by repeating the primary group comparisons including sex as a between-subjects factor. Given that this is the first study to characterize ICM in AUD, we elected to be comprehensive and report statistical significance at $p<.05$.

\section{Results}

\section{Sample Characteristics}

The AUD group ( $N=30)$ did not differ significantly from the control group $(N=33)$ with regard to sex, age, race, or handedness (Table 1). By nature of the case-control design, the AUD group had a higher AUDIT score and drinks per week. Compared to the AUD group, the control group reported higher education and higher median income. Finally, a greater percentage of participants in the AUD group reported weekly use of cannabis (although no participants met DSM-5 criteria for cannabis use disorder) or being a current cigarette smoker. 


\section{ICM Group Maps}

Surface visualization maps depicting ICM-related ratio signal for both the AUD and the control group are shown in Figure 2, Panels A-B. The pattern of anatomical distribution of ICM across groups was generally similar with the highest concentrations present throughout the visual cortex, primary motor and primary sensory regions, medial regions such as the anterior cingulate cortex (ACC), and the temporal pole. Visual comparison of the AUD and control maps suggests greater ICM-related MRI signal in the AUD group (indicated by darker or more extensive red areas in the maps), particularly in medial frontal regions in the left hemisphere, the cingulate gyrus in both hemispheres, and the anterior temporal lobe in the right hemisphere. Region of Interest Analysis

Mean ICM-related ratio signal was extracted from the 40 a priori ROIs and group differences were examined using ANOVA. Although there were significant differences in a number of potential covariates (see Table 1), none of these variables were significantly correlated with overall ICM in left or right hemisphere $(r s<.15)$ although the correlation between smoking status and right hemisphere ICM was marginally significant $(r=.25, p=.053)$. Thus, we elected to conduct the primary analyses with no covariates and then examine whether covarying for smoking impacted the statistical results.

The AUD group exhibited significantly increased ICM-related ratio signal in ten regions (see Figure 3 for ICM signal by group and Table 3 for statistical results). These included precuneus, ventromedial prefrontal cortex, posterior cingulate, and middle ACC in the left hemisphere; and middle/posterior insula, dorsolateral prefrontal cortex (Brodmann areas 8 and 46), posterior cingulate, temporal pole, and primary motor cortex in the right hemisphere. Of note, effect sizes (Cohen's d) for these significant group differences ranged from .50-.75, reflecting medium magnitude effects. When smoking was covaried, four of these effects remained significant, including precuneus, ventromedial prefrontal cortex and posterior cingulate in the left hemisphere and dorsolateral prefrontal cortex (BA8) in the right hemisphere. 


\section{Exploratory Whole-Brain Analysis}

We also characterized group differences outside of the a priori regions of interest (ROIs) by generating a whole-brain vertex-wise map of effect sizes (Cohen's d) (See Figure 2, Panel C). Here, the largest effect sizes (d's > .70) were located in the temporal pole, the ACC, and various medial and lateral frontal regions.

\section{Associations with Alcohol Problem Severity}

Bivariate correlations were conducted to examine whether ICM-related ratio signal was significantly associated with alcohol problem severity as assessed by the AUDIT total score. Significant positive correlations between greater ICM-related signal and greater alcohol problems were found for precuneus and middle ACC in the left hemisphere, and dorsolateral prefrontal cortex (BA8), posterior cingulate, temporal pole, and primary motor cortex in the right hemisphere.

\section{Sex Differences in ICM}

Sex differences in ICM-related MRI signal were examined by repeating the primary ANOVA models including sex at birth as an additional between-subjects factor. These analyses did not reveal any significant main effects of Sex (ps > .08) or Sex $\times$ Group interactions (ps > .11). Thus, the group differences observed above do not appear to be significantly impacted by participant sex.

\section{Discussion}

The current study is the first to use an optimized MRI pulse sequence to characterize ICM in a sample of participants with AUD and examine potential differences from a sample of healthy social drinkers. Previous research in healthy samples has reported the distribution of ICM across the cortex and provided initial clues about its role in neurocognitive processing. Prior studies with clinical samples have also indicated significant ICM disruption in people with bipolar disorder [16,24,25], schizophrenia [31,32], and other major mental health disorders [47]. 
Despite the increasing use of optimized MRI methods (e.g., [21]), the role of ICM in the pathophysiology of AUD remains unclear. Our results indicated that global distribution of ICM appears to be generally similar between people with AUD and controls; however, a finer-grained analysis among specific ROls reveals significant differences between groups with small-tomedium effect size magnitudes. In all cases, the AUD group exhibited significantly greater ICMrelated ratio signal compared to controls. These initial results provide important proof-of-concept for mapping ICM in vivo in people with AUD and, more importantly, offer a foundation for several future directions.

The primary finding was although ICM does not appear to be globally disrupted in the AUD group, it does appear to differ in specific ROls. Comparing the two groups, we found significantly higher ICM-related ratio signal in ten regions, including DLPFC, VMPFC, PCC, ACC, posterior insula, and precuneus, among others. When covarying for smoking status, differences in DLPFC, VMPFC, precuneus, and PCC remained significant. Another important finding was the small magnitude positive correlations between higher AUDIT score and greater ICM-related signal in DLPFC, ACC, PCC, precuneus, temporal pole, among other regions. Although we refrained from constructing hypotheses related to directionality, the finding that the AUD group had more ICM signal than the control participants was somewhat unexpected. Unfortunately, the cross-sectional design of this study and the relatively large number of participants who were currently engaged in alcohol treatment limit our ability to make inferences about why ICM was increased or whether differences would remain in participants who were not actively trying to reduce their drinking. Nevertheless, comparing the present results with previous ICM studies in healthy and clinical populations reveals some overlap in key regions. The PCC region in the current study is in the same general location as the Grydeland et al. study in healthy participants that reported associations between ICM in PCC and error processing[18]. Exploring whether increased ICM in PCC in people with AUD is related to 
neurocognitive performance is an important next step. Differences in DLPFC / Brodmann Area 46 have also been reported in previous studies with patients with bipolar disorder [25].

One potential explanation for our somewhat counterintuitive results may be that the increase in ICM signal is a compensatory response to neurotoxic effects or inflammatory responses to extended and excessive alcohol consumption. Alcohol has neurotoxic and inflammatory effects in both humans $[48,49]$ and rodents [50]. As well, neuroinflammation can lead to the initiation of myelin repair $[51,52]$, and oligodendrocyte precursor cells may differentiate into remyelinating oligodendrocytes when demyelination is present in the case of an injury or lesion [53]. Additionally, the biproducts of neuroinflammation, such as loss of tissue, demyelination, swelling (i.e., edema), can lead to various changes in T1 relaxation times [54] which may also be a possible explanation for the unexpected elevation in of ICM signal in the AUD group relative to controls. Importantly, much of this work is at the cellular level in animal models and should be interpreted with caution when translating findings to humans. However, it is reasonable to speculate that the increased ICM-related signal may stem from myelin-related compensatory mechanisms. As noted below, a priority for the future is to examine changes in ICM over time in people with AUD, while examining other systemic and central markers of inflammation (e.g., $[55,56])$.

Other priorities for future research include studying a larger sample of participants with a full range of alcohol involvement (e.g., across the continuum from social drinking through mild to severe AUD). As a proof-of-concept study, it was intentional to compare moderate/severe AUD with social drinkers, but a dimensional study will help to further characterize ICM variation in relation to AUD severity. Another important next step is to observe changes in ICM longitudinally which would not only address the question of when ICM-related differences emerge, but also what are the factors that relate to these differences such as timing of initiation of excessive drinking. Due to our cross-sectional design, we are unable to answer many compelling questions, such as whether ICM differences result from excessive alcohol use over 
time or whether ICM differences are present prior to onset of AUD (i.e., consequence or cause). Another important priority is to examine what happens to ICM when people reduce or stop alcohol consumption following treatment and whether individual differences in ICM at entry to treatment predict subsequent treatment outcomes (e.g., [3,57]).

Other limitations of our current study apart from the cross-sectional design include differences between the AUD and control participants; there were some differences between groups that should lead to some caution while interpreting the results. While some differences were deliberately due to the design (e.g., AUDIT score), there were significant differences in education, income, and proportion of participants who reported tobacco or cannabis use. Of these variables, only current smoking was associated with ICM and sensitivity analyses revealed that several differences remained even after controlling for smoking status.

In conclusion, this is the first study to our knowledge to examine ICM-related MRI signal in people diagnosed with AUD. The distribution of ICM across the cortex was largely similar between the AUD and control groups, although a more precise analysis revealed several areas of greater ICM signal in the AUD participants. These initial findings offer proof-of-concept for studying ICM in addiction samples and provide a foundation for future studies to unpack the clinical and neurocognitive significance of the differences observed, neurobiological mechanisms (e.g., compensatory or neuroinflammatory changes), and potential for normalization of ICM following treatment. 


\section{Funding and Disclosures}

This work was funded by a grant from the National Institute on Alcohol Abuse and Alcoholism (NIAAA R21AA026392 to M.A.) and the Peter Boris Center for Addictions Research at McMaster University (M.A.). J.M. holds the Peter Boris Chair in Addictions Research which partially supported his role. James MacKillop is a principal in BEAM Diagnostics Inc, but no BEAM products were used in this study. No other authors have conflicts of interest to declare.

\section{Acknowledgements}

We dedicate this paper to the memory of Richard Filc, BSc (Honours), McMaster University, who was a founding undergraduate researcher in the Cognitive Neuroscience of Addictions Laboratory and a passionate advocate for addictions science and recovery. We thank the team members of the Cognitive Neuroscience of Addictions Laboratory at McMaster University for contributions to data collection and data processing, especially Lana Vedelago, MSc, and Emma Marsden. We also thank Christopher Rowley, PhD, and Laagishan Yoganathan, MSc, for assistance with data analysis. We thank Norm Konyer and the staff of the Imaging Research Center at St. Joseph's Healthcare Hamilton for assistance with MRI scanning. The authors recognize and acknowledge that this work was conducted on the traditional territories of the Mississauga and Haudenosaunee nations, and within the lands protected by the Dish With One Spoon wampum agreement.

\section{Author Contributions}

MA, NB, LM, and JM were responsible for study concept and design. VM collected data with oversight from MA. VM and MA conducted the data analyses with input and interpretation from NB and JM. VM and MA wrote the first draft of the manuscript. All authors read and provided edits to the manuscript and approved the final version for submission. 


\section{References}

1. Sullivan E V., Harris RA, Pfefferbaum A. Alcohol's effects on brain and behavior. Alcohol Res Heal. 2010;33:127-143.

2. Fritz M, Klawonn AM, Zahr NM. Neuroimaging in alcohol use disorder: From mouse to man. J Neurosci Res. 2019:1-19.

3. Durazzo TC, Tosun D, Buckley S, Gazdzinski S, Mon A, Fryer SL, et al. Cortical thickness, surface area, and volume of the brain reward system in alcohol dependence: relationships to relapse and extended abstinence. Alcohol Clin Exp Res. 2011;35:11871200.

4. Fortier CB, Leritz EC, Salat DH, Venne JR, Maksimovskiy AL, Williams V, et al. Reduced cortical thickness in abstinent alcoholics and association with alcoholic behavior. Alcohol Clin Exp Res. 2011;35:2193-2201.

5. Bühler M, Mann K. Alcohol and the human brain: a systematic review of different neuroimaging methods. Alcohol Clin Exp Res. 2011;35:1771-1793.

6. Momenan R, Grodin EN. Decreased Subcortical Volumes in Alcohol Dependent Individuals: Effect of Polysubstance Use Disorder. Addict Biol. 2017;22:1426-1437.

7. Yang X, Tian F, Zhang H, Zeng J, Chen T, Wang S, et al. Cortical and subcortical gray matter shrinkage in alcohol-use disorders: A voxel-based meta-analysis. Neurosci Biobehav Rev. 2016;66:92-103.

8. Mashhoon Y, Czerkawski C, Crowley DJ, Cohen-Gillbert JE, Sneider JT, Silveri MM. Binge Alcohol Consumption in Emerging Adults: Anterior Cingulate Cortical "Thinness" Is Associated with Alcohol Use Patterns. Alcohol Clin Exp Res. 2014. 2014. https://doi.org/10.1111/acer.12475.

9. Pennington DL, Durazzo TC, Schmidt TP, Abé C, Mon A, Meyerhoff DJ. Alcohol use disorder with and without stimulant use: brain morphometry and its associations with cigarette smoking, cognition, and inhibitory control. PLoS One. 2015;10:e0122505. 
10. Arain M, Haque M, Johal L, Mathur P, Nel W, Rais A, et al. Maturation of the adolescent brain. Neuropsychiatr Dis Treat. 2013;9:449-461.

11. Haroutunian V, Katsel P, Roussos P, Davis KL, Altshuler LL, Bartzokis G. Myelination, oligodendrocytes, and serious mental illness. Glia. 2014;62:1856-1877.

12. Bartzokis G. Alzheimer's disease as homeostatic responses to age-related myelin breakdown. Neurobiol Aging. 2011;32:1341-1371.

13. Sowell ER, Peterson BS, Thompson PM, Welcome SE, Henkenius AL, Toga AW. Mapping cortical change across the human life span. Nat Neurosci. 2003;6:309-315.

14. Filley CM, McConnell B V., Anderson CA. The expanding prominence of toxic leukoencephalopathy. J Neuropsychiatry Clin Neurosci. 2017;29:308-318.

15. Nieuwenhuys R. The myeloarchitectonic studies on the human cerebral cortex of the Vogt-Vogt school, and their significance for the interpretation of functional neuroimaging data. Brain Struct Funct. 2013.

16. Rowley CD, Bazin P-L, Tardif CL, Sehmbi M, Hashim E, Zaharieva N, et al. Assessing intracortical myelin in the living human brain using myelinated cortical thickness. Front Neurosci. 2015;9:396.

17. Unterrainer HF, Hiebler-Ragger M, Koschutnig K, Fuchshuber J, Ragger K, Perchtold CM, et al. Brain Structure Alterations in Poly-Drug Use: Reduced Cortical Thickness and White Matter Impairments in Regions Associated With Affective, Cognitive, and Motor Functions. Front Psychiatry. 2019;10:1-10.

18. Grydeland H, Westlye LT, Walhovd KB, Fjell AM. Intracortical Posterior Cingulate Myelin Content Relates to Error Processing: Results from T1- and T2-Weighted MRI Myelin Mapping and Electrophysiology in Healthy Adults. Cereb Cortex. 2015:bhv065-.

19. Grydeland H, Walhovd KB, Tamnes CK, Westlye LT, Fjell AM. Intracortical myelin links with performance variability across the human lifespan: results from T1- and T2-weighted MRI myelin mapping and diffusion tensor imaging. J Neurosci. 2013;33:18618-18630. 
20. Glasser MF, Van Essen DC. Mapping human cortical areas in vivo based on myelin content as revealed by T1- and T2-weighted MRI. J Neurosci. 2011;31:11597-11616.

21. Bock NA, Hashim E, Janik R, Konyer NB, Weiss M, Stanisz GJ, et al. Optimizing T1weighted imaging of cortical myelin content at 3.0 T. Neuroimage. 2013;65:1-12.

22. Bock NA, Kocharyan A, Liu J V, Silva AC. Visualizing the entire cortical myelination pattern in marmosets with magnetic resonance imaging. J Neurosci Methods. $2009 ; 185: 15-22$.

23. Fracasso A, Van Veluw SJ, Visser F, Luijten PR, Spliet W, Zwanenburg JJM, et al. Lines of Baillarger in vivo and ex vivo: Myelin contrast across lamina at $7 \mathrm{~T} \mathrm{MRI}$ and histology. Neuroimage. 2016;133:163-175.

24. Sehmbi M, Rowley CD, Minuzzi L, Kapczinski F, Kwiecien JM, Bock NA, et al. Agerelated deficits in intracortical myelination in young adults with bipolar disorder type I. J Psychiatry Neurosci. 2019;44:79-88.

25. Sehmbi M, Rowley CD, Minuzzi L, Kapczinski F, Steiner M, Sassi RB, et al. Association of intracortical myelin and cognitive function in bipolar I disorder. Acta Psychiatr Scand. 2018;138:62-72.

26. Miller DJ, Duka T, Stimpson CD, Schapiro SJ, Baze WB, McArthur MJ, et al. Prolonged myelination in human neocortical evolution. Proc Natl Acad Sci U S A. 2012;109:1648016485.

27. Öngür D, Drevets WC, Price JL. Glial reduction in the subgenual prefrontal cortex in mood disorders. Proc Natl Acad Sci U S A. 1998;95:13290-13295.

28. Uranova NA, Vostrikov VM, Orlovskaya DD, Rachmanova VI. Oligodendroglial density in the prefrontal cortex in schizophrenia and mood disorders: A study from the Stanley Neuropathology Consortium. Schizophr Res. 2004;67:269-275.

29. Lewandowski KE, Ongür D, Sperry SH, Cohen BM, Sehovic S, Goldbach JR, et al. Myelin vs Axon Abnormalities in White Matter in Bipolar Disorder. 
Neuropsychopharmacology. 2015;40:1243-1249.

30. Tkachev D, Mimmack ML, Ryan MM, Wayland M, Freeman T, Jones PB, et al.

Oligodendrocyte dysfunction in schizophrenia and bipolar disorder. Lancet. 2003;362:798-805.

31. Bartzokis G, Lu PH, Raven EP, Amar CP, Detore NR, Couvrette AJ, et al. Impact on intracortical myelination trajectory of long acting injection versus oral risperidone in firstepisode schizophrenia. Schizophr Res. 2012. 2012.

https://doi.org/10.1016/j.schres.2012.06.036.

32. Tishler TA, Bartzokis G, Lu PH, Raven EP, Khanoyan M, Kirkpatrick CJ, et al. Abnormal Trajectory of Intracortical Myelination in Schizophrenia Implicates White Matter in Disease Pathophysiology and the Therapeutic Mechanism of Action of Antipsychotics. Biol Psychiatry Cogn Neurosci Neuroimaging. 2018;3:454-462.

33. Cantacorps L, Alfonso-Loeches S, Moscoso-Castro M, Cuitavi J, Gracia-Rubio I, LópezArnau R, et al. Maternal alcohol binge drinking induces persistent neuroinflammation associated with myelin damage and behavioural dysfunctions in offspring mice. Neuropharmacology. 2017;123:368-384.

34. Glasser MF, Sotiropoulos SN, Wilson JA, Coalson TS, Fischl B, Andersson JL, et al. The minimal preprocessing pipelines for the Human Connectome Project. Neuroimage. 2013;80:105-124.

35. Saunders JB, Aasland OG, Babor TF, de la Fuente JR, Grant M. Development of the Alcohol Use Disorders Identification Test (AUDIT): WHO collaborative project on early detection of persons with harmful alcohol consumption. Il. Addiction. 1993;88:791-804.

36. Koob GF, Volkow ND. Neurocircuitry of Addiction. Neuropsychopharmacology. 2010;35:217-238.

37. Crews FT, Boettiger CA. Impulsivity, frontal lobes and risk for addiction. Pharmacol Biochem Behav. 2009;93:237-247. 
38. Longo DL, Volkow ND, Koob GF, McLellan AT. Neurobiologic Advances from the Brain Disease Model of Addiction. N Engl J Med. 2016;374:363-371.

39. Momenan R, Steckler LE, Saad ZS, van Rafelghem S, Kerich MJ, Hommer DW. Effects of alcohol dependence on cortical thickness as determined by magnetic resonance imaging. Psychiatry Res. 2012. 2012. https://doi.org/10.1016/j.pscychresns.2012.05.003.

40. Lawyer G, Bjerkan PS, Hammarberg A, Jayaram-Lindström N, Franck J, Agartz I. Amphetamine dependence and co-morbid alcohol abuse: associations to brain cortical thickness. BMC Pharmacol. 2010. 2010. https://doi.org/10.1186/1471-2210-10-5.

41. Morris VL, Owens MM, Syan SK, Petker TD, Sweet LH, Oshri A, et al. Associations Between Drinking and Cortical Thickness in Younger Adult Drinkers: Findings From the Human Connectome Project. Alcohol Clin Exp Res. 2019;43:1918-1927.

42. McHugh RK, Votaw V, Sugarman D, Greenfield S. Sex and Gender Differences in Substance Use Disorders. Clin Psychol Rev. 2018:12-23.

43. First MB, Williams JBW, Karg RS, Spitzer RL. Structured Clinical Interview for DSM-5 Disorders - Clinician Version (SCID-5-CV). 2015.

44. Sobell LC, Sobell MB. Timeline follow-back: A technique for assessing self-reported alcohol consumption. In: Litten RZ, Allen JP, editors. Meas. Alcohol Consum. Psychosoc. Biochem. Methods., Totowa, NJ: Humana Press; 1992. p. 41-72.

45. Heatherton TF, Kozlowski LT, Frecker RC, Fagerstrom KO. The Fagerstrom Test for Nicotine Dependence: A revision of the Fagerstrom Tolerance Questionnaire. Br J Addict. 1991;86:1119-1127.

46. Glasser MF, Coalson TS, Robinson EC, Hacker CD, Yacoub E, Ugurbil K, et al. A multimodal parcellation of human cerebral cortex. Nature. 2017;536:171-178.

47. Luo X, Li K, Zeng Q, Huang P, Jiaerken Y, Wang S, et al. Application of T1-/T2-Weighted Ratio Mapping to Elucidate Intracortical Demyelination Process in the Alzheimer's Disease Continuum. Front Neurosci. 2019;13:1-13. 
48. Wang HJ, Zakhari S, Jung MK. Alcohol, inflammation, and gut-liver-brain interactions in tissue damage and disease development. World J Gastroenterol. 2010;16:1304-1313.

49. Leclercq S, De Timary P, Delzenne NM, Stärkel P. The link between inflammation, bugs, the intestine and the brain in alcohol dependence. Transl Psychiatry. 2017;7.

50. Pascual M, Pla A, Miñarro J, Guerri C. Neuroimmune activation and myelin changes in adolescent rats exposed to high-dose alcohol and associated cognitive dysfunction: A review with reference to human adolescent drinking. Alcohol Alcohol. 2014.

51. Tang Y, Le W. Differential Roles of M1 and M2 Microglia in Neurodegenerative Diseases. Mol Neurobiol. 2016;53:1181-1194.

52. Glezer I, Simard AR, Rivest S. Neuroprotective role of the innate immune system by microglia. Neuroscience. 2007;147:867-883.

53. Setzu A, Lathia J, Zhao C, Wells K, Rao M, French-Constant C, et al. Inflammation Stimulates Myelination by Transplanted Oligodendrocyte Precursor Cells. Glia. 2006:297-303.

54. Albrecht DS, Granziera C, Hooker JM, Loggia ML. In Vivo Imaging of Human Neuroinflammation. ACS Chem Neurosci. 2016;7:470-483.

55. de Timary P, Stärkel P, Delzenne NM, Leclercq S. A role for the peripheral immune system in the development of alcohol use disorders? Neuropharmacology. 2017;122:148-160.

56. García-Baos A, Alegre-Zurano L, Cantacorps L, Martín-Sánchez A, Valverde O. Role of cannabinoids in alcohol-induced neuroinflammation. Prog Neuro-Psychopharmacology Biol Psychiatry. 2021;104.

57. Cardenas VA, Durazzo TC, Gazdzinski S, Mon A, Studholme C, Meyerhoff DJ. Brain morphology at entry into treatment for alcohol dependence is related to relapse propensity. Biol Psychiatry. 2011. 2011. https://doi.org/10.1016/j.biopsych.2011.04.003. 


\section{Figure Legends}

Figure 1. Anatomical locations of a priori regions of interest generated from the multimodial parcellation atlas. A total of 20 ROls were examined in each hemisphere. Region numbers correspond to numeric labels in Table 2, and full list of MMP regions comprising each ROI are provided in Supplementary Table 1.

Figure 2. Group average ICM maps for AUD (top) and control (middle) participants. Bottom panel depicts vertex-wise effect size maps (Cohen's d) reflecting differences between AUD and controls. In each panel data are projected onto the middle-depth cortical surface, shown in lateral, medial and superior views for left and right hemispheres separately.

Figure 3. Extracted ICM-related ratio signal values for each a priori ROI for left hemisphere (Panel A) and right hemisphere (Panel B). AUD group shown in black; control group shown in gray. Bars reflect mean +1 standard error. ${ }^{*} p<.05$. Region abbreviations provided in Table 2. 
Table 1. Sample Characteristics

\begin{tabular}{|c|c|c|c|}
\hline Variable & AUD (N = 30) & $\operatorname{CON}(N=33)$ & $p$ \\
\hline Age & $39.63(9.65)$ & $36.93(10.46)$ & .29 \\
\hline Sex & $33 \%$ Female & $58 \%$ Female & .06 \\
\hline Race & $86 \%$ White & $84 \%$ White & .67 \\
\hline Education & $14.43(2.62)$ & $17.45(2.58)$ & $<.001$ \\
\hline Income (Median) & $\$ 30-45,000$ & $\$ 60-75,000$ & $<.001$ \\
\hline DSM-5 AUD Severity & $\begin{array}{l}3 \% \text { Moderate } \\
97 \% \text { Severe }\end{array}$ & $N / A$ & \\
\hline AUDIT & $31.36(5.50)$ & $3.84(1.75)$ & $<.001$ \\
\hline Drinks/Week & $16.06(24.35)$ & $3.06(1.98)$ & .003 \\
\hline Weekly Cannabis & $23 \%$ & $6 \%$ & .007 \\
\hline Current Smoker & $50 \%$ & $6 \%$ & $<.001$ \\
\hline FTND Total (smokers) & $4.53(2.67)$ & $2.33(2.08)$ & .20 \\
\hline Handedness & $80 \%$ Right & 84\% Right & .90 \\
\hline
\end{tabular}

Note: AUD = alcohol use disorder; CON = control; DSM-5 = Diagnostic and Statistical Manual of Mental Disorders, $5^{\text {th }}$ Edition; AUDIT = Alcohol Use Disorders Identification Test; FTND = Fagerstrom Test for Nicotine Dependence 
Table 2. Region of Interest Analysis and Associations with Alcohol Severity

\begin{tabular}{|c|c|c|c|c|c|c|c|c|c|c|c|}
\hline \multirow[b]{2}{*}{ \# } & \multirow[b]{2}{*}{ Region } & \multicolumn{4}{|c|}{ Left Hemisphere } & \multicolumn{4}{|c|}{ Right Hemisphere } & \multicolumn{2}{|c|}{ AUDIT Total } \\
\hline & & $F$ & $P$ & $n p^{2}$ & d & $F$ & $P$ & $n p^{2}$ & d & $r$ & $\mathrm{r}$ \\
\hline$\overline{1}$ & Anterior Insula & 2.50 & 0.119 & 0.039 & 0.40 & 3.83 & 0.055 & 0.059 & 0.49 & .223 & .224 \\
\hline 3 & Precuneus & 4.66 & 0.035 & 0.071 & 0.54 & 2.65 & 0.108 & 0.042 & 0.41 & $.260^{*}$ & .204 \\
\hline 4 & Primary Motor (M1) & 1.15 & 0.288 & 0.019 & 0.27 & 5.59 & 0.021 & 0.084 & 0.59 & .159 & $.329 * *$ \\
\hline 5 & IFG & 2.56 & 0.115 & 0.040 & 0.40 & 1.36 & 0.249 & 0.022 & 0.29 & .203 & .156 \\
\hline 8 & DLPFC (BA46) & 3.16 & 0.080 & 0.049 & 0.45 & 4.25 & 0.044 & 0.065 & 0.52 & .234 & .225 \\
\hline 9 & Medial PFC (BA10) & 0.74 & 0.394 & 0.012 & 0.21 & 0.80 & 0.373 & 0.013 & 0.22 & .114 & .150 \\
\hline 10 & Medial PFC (BA8) & 3.23 & 0.077 & 0.050 & 0.45 & 1.57 & 0.216 & 0.025 & 0.31 & .201 & .190 \\
\hline 11 & Medial PFC (BA9) & 2.20 & 0.143 & 0.035 & 0.37 & 1.22 & 0.274 & 0.020 & 0.28 & .186 & .151 \\
\hline 12 & VMPFC & 4.27 & 0.043 & 0.065 & 0.52 & 2.60 & 0.112 & 0.041 & 0.40 & .235 & .219 \\
\hline 17 & STG & 1.28 & 0.262 & 0.021 & 0.28 & 3.25 & 0.077 & 0.051 & 0.45 & .156 & .235 \\
\hline 18 & MTG & 0.55 & 0.461 & 0.009 & 0.19 & 3.22 & 0.078 & 0.050 & 0.45 & .129 & .226 \\
\hline 19 & ITG & 0.36 & 0.552 & 0.006 & 0.15 & 0.95 & 0.334 & 0.015 & 0.24 & .092 & .119 \\
\hline 20 & Temporal Pole & 2.74 & 0.103 & 0.043 & 0.41 & 6.01 & 0.017 & 0.090 & 0.61 & .244 & $.285^{\star}$ \\
\hline
\end{tabular}

Note: ${ }^{*} \mathrm{p}<.05 ;{ }^{* *} \mathrm{p}<.01 ; \mathrm{IFG}=$ inferior frontal gyrus; DLPFC = dorsolateral prefrontal cortex; PFC = prefrontal cortex; BA = Brodmann area; VMPFC = ventromedial prefrontal cortex; $P C C=$ posterior cingulate cortex; $A C C=$ anterior cingulate cortex; $S T G=$ superior temporal gyrus; $\mathrm{MTG}=$ middle temporal gyrus; ITG = inferior temporal gyrus; AUDIT = Alcohol Use Disorders Identification Test 
Figure 1.

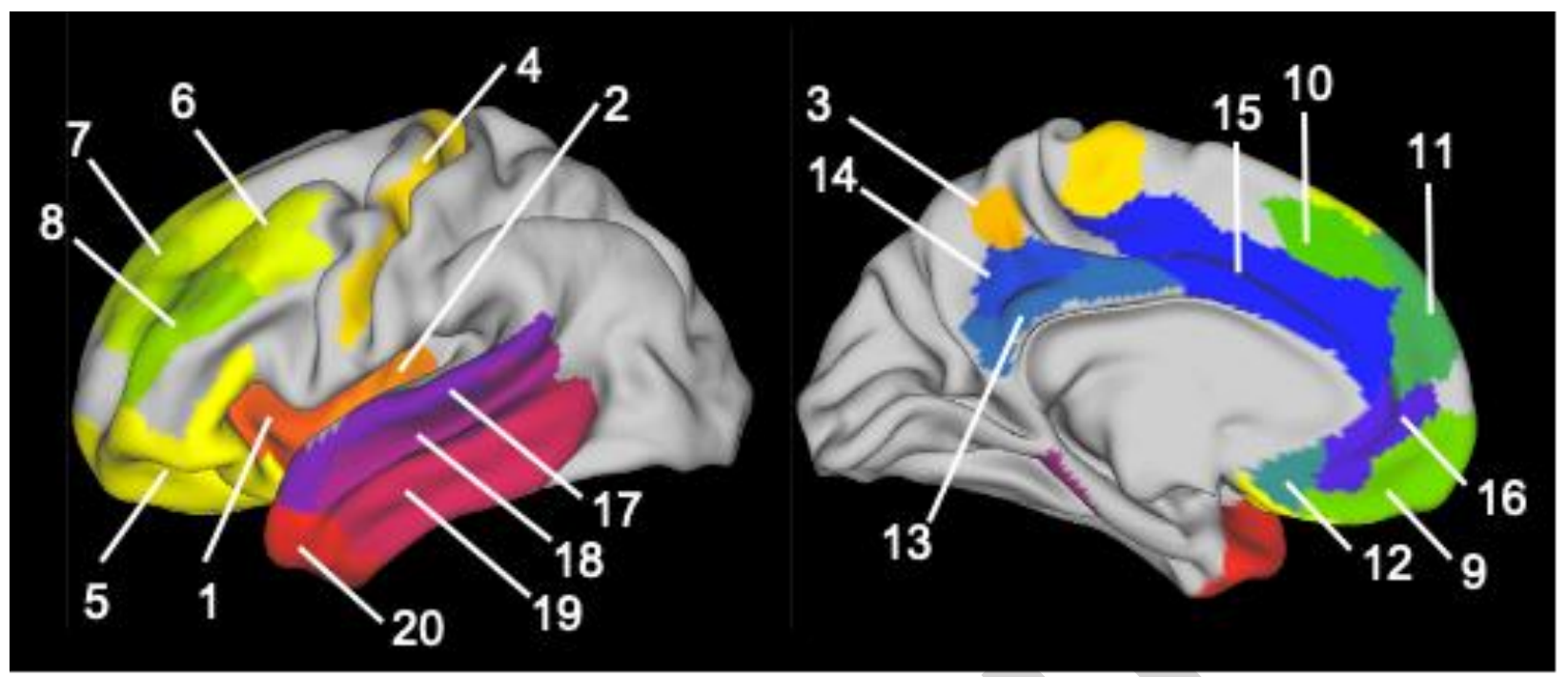


Figure 2.

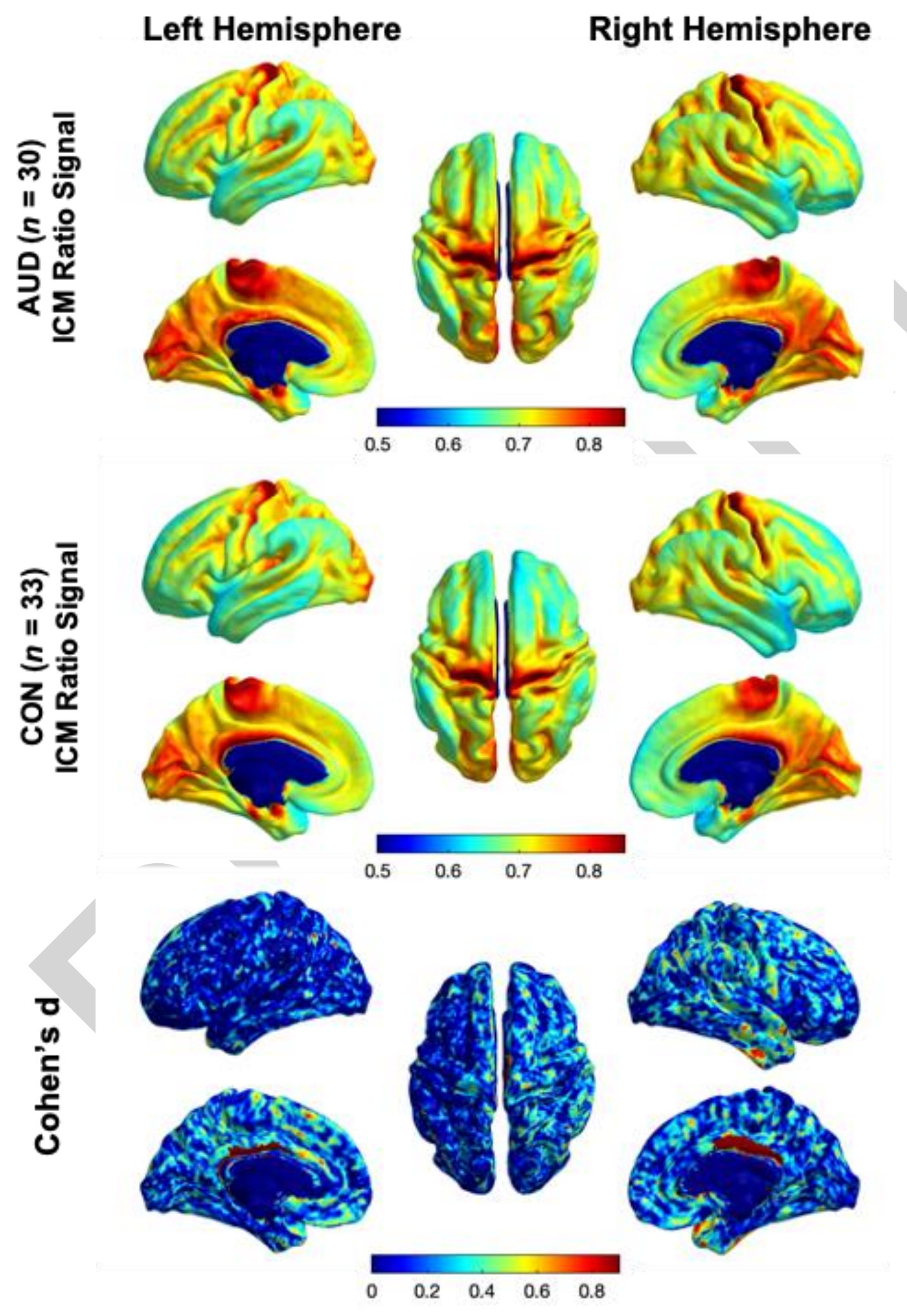


Figure 3.

A) Left Hemisphere

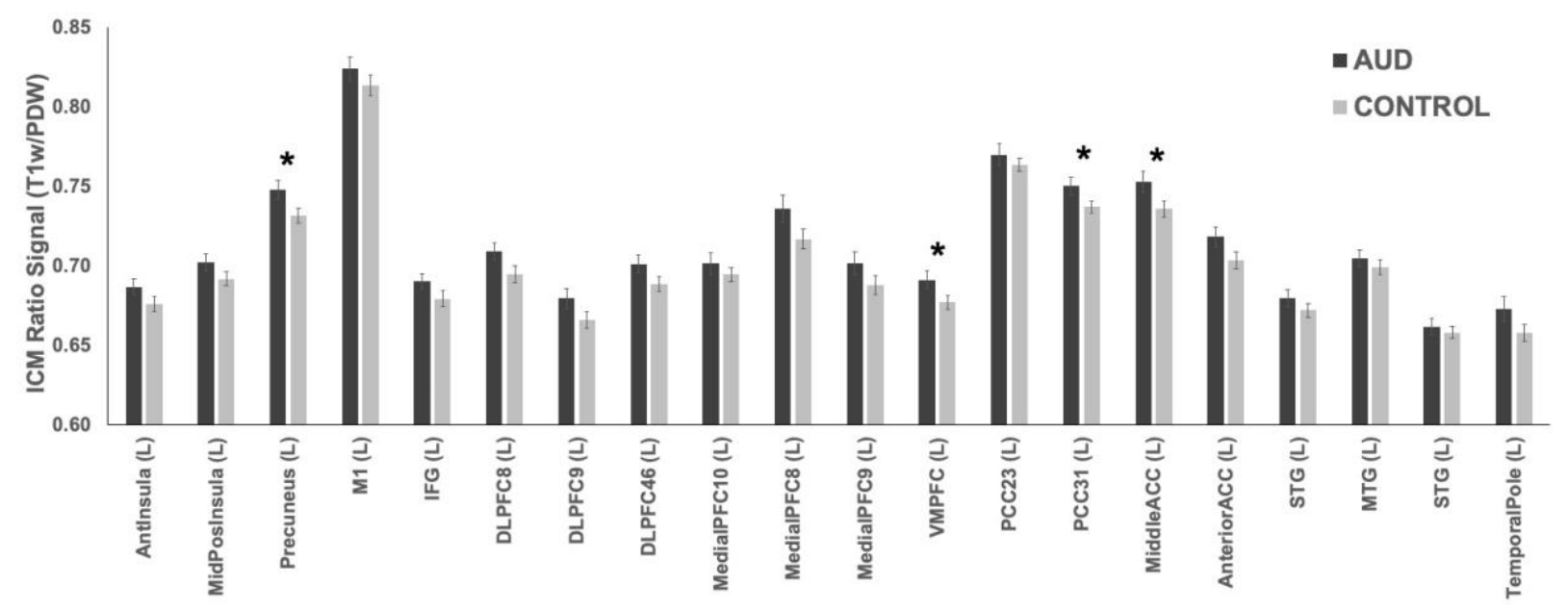

B) Right Hemisphere

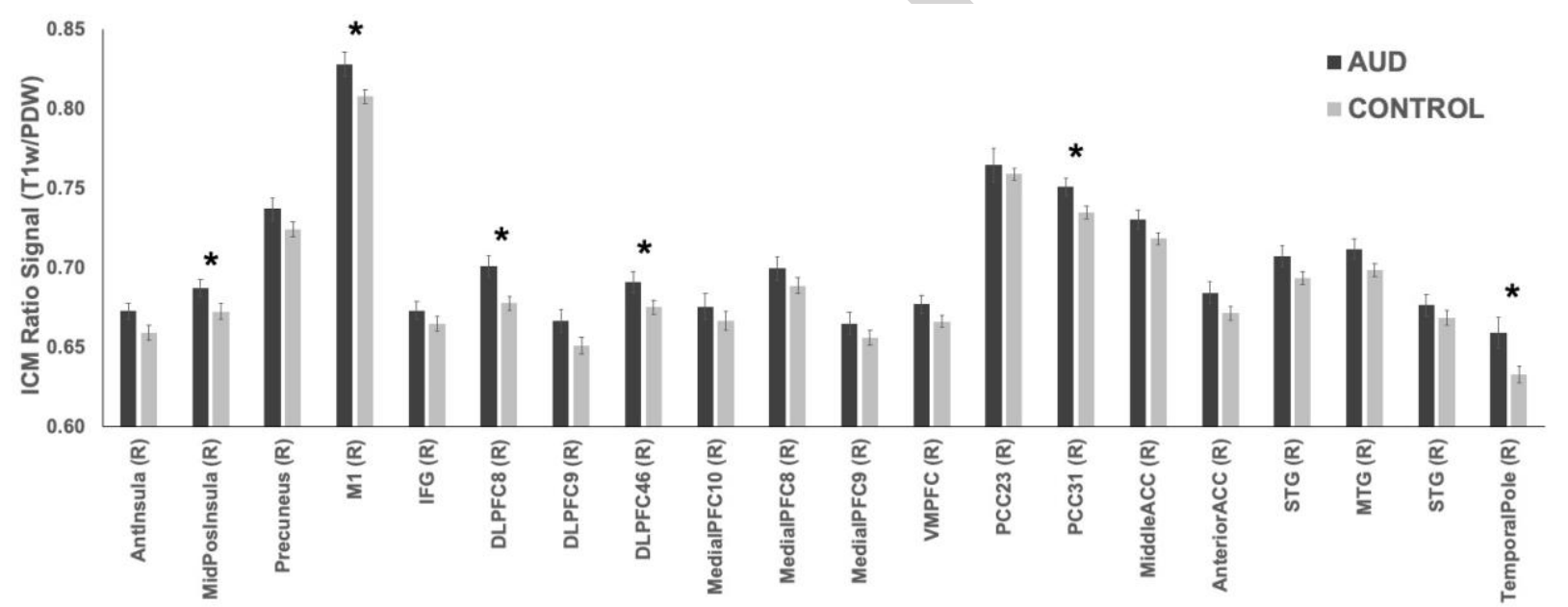




\section{Supplementary Materials}

\section{Supplementary Methods}

MRI Scanning Protocol

For our anatomical reference, we acquired a 3D T1-weighted anatomical image of the whole head using a 3D inversion-recovery gradient-echo sequence (GE 3D BRAVO; Inversion time $(\mathrm{TI})=450 \mathrm{~ms}, \mathrm{TE}=3.2 \mathrm{~ms}, \mathrm{TR}=58.4 \mathrm{~ms}$, flip angle $=12^{\circ}$, field of view $(F O V)=25.6 \times 25.6 \times 25.6 \mathrm{~cm})$. This image was used as a reference for all registration steps. We also acquired two T1-weighted images with high intracortical contrast (one per hemisphere) using an inversion-recovery gradient-echo sequence (GE 3D BRAVO; TI=1000ms; TR=8.4ms; TE=3.2ms; flip angle=12 $; F O V=24 \times 10 \times 24 \mathrm{~cm}$; Matrix $=240 \times 100 \times 240 \mathrm{~cm}$; time between end of acquisition block and next $180^{\circ}$ pulse (TD) $=1100 \mathrm{~ms}$; centric phase encoding) that was used in the creation of the ICM maps. Each hemisphere was imaged separately, and the resulting images were stitched together to form a complete anatomical image. Lastly, we collected a 3D proton density-weighted image of the whole head (GE 3D FSPGR; TR=7.9ms, TE=3.1ms, flip angle $=4^{\circ}, \mathrm{FOV}=24 \times 17 \times 24 \mathrm{~cm}$ ) to normalize intensity inhomogeneities and remove $\mathrm{T}^{*}$-weighting in the high intracortical contrast T1-weighted image.

Image Preprocessing and ICM Calculations

Raw DICOM image files were assembled into NIFTI datasets using MRIcroGL's dcm2nii. To account for head motion between scans, all images were registered to the $T_{1}$-weighted image with high intracortical contrast using a 6-parameter linear affine registration using FLIRT. All images ( $T_{1}, T_{1}$ with high intracortical contrast, proton density-weighted) were then registered to the $\mathrm{MNI} 152 \mathrm{~T}_{1} 1 \mathrm{~mm}$ brain atlas. The resulting image was filtered using a $5 \times 5 \times 5 \mathrm{~mm} 3 \mathrm{D}$ median filter. A strongly $T_{1}$-weighted ratio image was created by dividing the $T_{1}$-weighted high intracortical contrast image by the filtered proton-density-weighted image. This removed radiofrequency receive field $\left(B_{1}-\right)$ inhomogeneities, some radiofrequency transmit field $\left(B_{1}+\right)$ inhomogeneities, and $\mathrm{T}_{2}{ }^{*}$-weighting arising from the gradient echo readout in the inversion- 
recovery image. The ratio image still contained some $B_{1}+$ inhomogeneity from the magnetization preparation portion of the inversion-recovery pulse sequence. Freesurfer segmentation was performed using recon-all, with manual corrections for gray matter / pial boundary errors. The remaining processing steps closely followed the Human Connectome Project's minimal processing pipeline using custom scripts for myelin mapping adapted from the HCP scripts [34] (https://github.com/Washington-University/HCPpipelines). These scripts use the output of Freesurfer's recon-all to generate myelin maps from the image data. Surface data was then brought into MATLAB using gifti tool box and ICM surface visualizations were created using the SurfStat toolbox.

\section{Supplementary Table 1. Regions of Interest from Multimodal Parcellation Atlas}

\begin{tabular}{lll}
\hline$\#$ & Region & MMP \#s \\
\hline 1 & Insula (anterior) & $109,111,112$ \\
2 & Insula (middle/posterior) & $106,167,168$ \\
3 & Precuneus & 27 \\
4 & Primary motor cortex (M1) & 8 \\
5 & Inferior frontal gyrus / orbitofrontal cortex & $66,75,76,77,90,91,92,93,94,166$ \\
6 & DLPFC (BA8) & $67,68,70,73$ \\
7 & DLPFC (BA9) & 71,87 \\
8 & DLPFC (BA46) & $83,84,85,86$ \\
9 & Medial frontal gyrus (BA10) & 65,88 \\
10 & Medial frontal gyrus (BA8) & 63 \\
11 & Medial frontal gyrus (BA9) & 69 \\
12 & Ventromedial PFC & 164 \\
13 & Posterior cingulate (BA23) & $32,33,34$ \\
14 & Posterior cingulate (BA31) & $35,161,162$ \\
15 & Anterior / Middle cingulate & $41,57,58,59,60,62,179,180$ \\
16 & Anterior cingulate & $61,64,165$ \\
17 & Superior temporal & $123,125,172,28$ \\
18 & Middle temporal & $126,128,129,130,139,176$ \\
19 & Inferior temporal & $132,133,134,137,177$ \\
20 & Temporal pole & 131,172 \\
\hline
\end{tabular}

